Submitted to The Astrophysical Journal

\title{
PROMPT Observations of the Early-Time Optical Afterglow of GRB 060607A
}

\author{
M. Nysewander ${ }^{12}$, D. E. Reichart ${ }^{1}$, J. A. Crain ${ }^{1}$, A. Foster ${ }^{1}$, J. Haislip ${ }^{1}$, K. Ivarsen ${ }^{1}$, A. \\ Lacluyze $^{1}$, A. Trotter ${ }^{1}$.
}

\begin{abstract}
PROMPT (Panchromatic Robotic Optical Monitoring and Polarimetry Telescopes) observed the early-time optical afterglow of GRB 060607A and obtained a densely sampled multiwavelength light curve that begins only tens of seconds after the GRB. Located at Cerro Tololo Inter-American Observatory in Chile, PROMPT is designed to observe the afterglows of $\gamma$-ray bursts using multiple automated $0.4-\mathrm{m}$ telescopes that image simultaneously in many filters when the afterglow is bright and may be highly variable. The data span the interval from 44 seconds after the GRB trigger to 3.3 hours in the Bgri filters. We observe an initial peak in the light curve at approximately three minutes, followed by rebrightenings peaking around 40 minutes and again at 66 minutes. Although our data overlap with the early Swift $\gamma$-ray and x-ray light curves, we do not see a correlation between the optical and high-energy flares. We do not find evidence for spectral evolution throughout the observations. We model the variations in the light curves and find that the most likely cause of the rebrightening episodes is a refreshment of the forward shock preceded by a rapidly fading reverse shock component, although other explanations are plausible.
\end{abstract}

Subject headings: gamma rays: bursts

\footnotetext{
${ }^{1}$ Department of Physics and Astronomy, University of North Carolina at Chapel Hill, Campus Box 3255 , Chapel Hill, NC 27599

${ }^{2}$ Space Telescope Science Institute, 3700 San Martin Drive, Baltimore, MD, 21218; mnysewan@stsci.edu
} 


\section{Introduction}

The general behavior of the broadband spectra and light curves of gamma-ray burst (GRB) afterglows has been shown to be well described by a jetted, relativistically expanding shell colliding with an external medium, which has been described as either that of a constant density (Sari et al. 1998) or wind-swept environment (Chevalier \& Li 2000). Since the discovery of the first x-ray, optical and radio afterglows over a decade ago (Costa et al. 1997; van Paradijs et al. 1997; Frail et al. 1997) astronomers have successfully applied these models to derive the conditions of the shock and the properties of the surrounding medium. In the past decade, the number and quality of afterglow follow-up observations have improved dramatically. The average response time for a GRB is now minutes instead of days, and for this rapid follow up, astronomers regularly use medium and large-aperture telescopes that can reach deep limiting magnitudes.

However, in nearly all of the most densely sampled, high signal-to-noise light curves, significant variations are seen super-imposed upon the general behavior. Three main physical scenarios have been proposed to explain these features: (1) the "patchy shell" model (Kumar \& Piran 2000a), (2) delayed shocks or energy injections from a long-lived central engine (Rees \& Meszaros 1998; Kumar \& Piran 2000b; Sari \& Mészáros 2000), and (3) variations in the circumburst density (Wang \& Loeb 2000; Dai \& Lu 2002). Although deviations from the simple power-law decay have been observed since the beginning of the afterglow era (GRB 970508; Panaitescu et al. 1998), the bright afterglows of GRB 021004 and GRB 030329 provide some of the most densely sampled light curves, and both have been used as test cases for these three mechanisms.

In the patchy shell model, random variations in the energy per unit angle in the outflow create the observed bumps in the light curve; the amplitude of these bumps is expected to decrease with time. Nakar et al. (2003) find that this model is slightly preferred over the other two for GRB 021004 because of the morphology of the fluctuations and a consistent value for the electron index between low and high energies. de Ugarte Postigo et al. (2005) also find this to be an acceptable scenario in their detailed analysis of the broadband (optical, near-infrared and millimeter) afterglow light curve. However, for GRB 030329 the patchy shell model has been ruled out because the variations occurred after the jet break, when contributions from this effect ought to be negligible (Granot et al. 2003).

Energy injections can be described as either delayed shocks that are hypothesized to be slower moving shells emitted by the central engine that catch up and impact the main shell of the forward shock as it decelerates (Zhang \& Mészáros 2002), or the result of a long-lived central engine. In this scenario, the light curve rises briefly as a result of the injection after which it resumes its decay with an index similar to the previous index, but with a 
new normalization that reflects the larger energy of the shock. Typically, the result of an energy injection is an overall upward shift of the afterglow light curve. Additionally, when the impact occurs, it may also produce a bright reverse shock that propogates backwards through the ejecta in the comoving frame (Nakar \& Piran 2003). This scenario was first proposed for GRB 021004 by Fox et al. (2003) and GRB 030329 by Granot et al. (2003). For GRB 021004, both Björnsson et al. (2004) and de Ugarte Postigo et al. (2005) find that the light curves, broadband spectral evolution and polarization signature of GRB 021004 can be well-modeled with a series of energy injections. Due to the fact that the rebrightening episodes in the light curve of GRB 030329 appear to be simple re-normalizations, both Granot et al. (2003) and Huang et al. (2006) find refreshed shocks to be the most natural explanation of the event.

Shocks resulting from the impact of the fireball upon density enhancements in the surrounding medium can cause significant rebrightenings in the GRB afterglow. When the shockwave hits a higher density, the flux sharply increases before it fades to match a light curve that is described by the higher density, however, if the density jump is large enough, it may also produce a reverse shock. Lazzati et al. (2002) propose that the variations in the light curve of GRB 021004 are likely due to moderate changes in density that only slightly modify the dynamics of the fireball. Many groups find that density variations can not account for the light curve of GRB 030329 due to the increase in flux normalization after each episode (Granot et al. 2003; Huang et al. 2006; Uemura et al. 2004).

Early multi-color light curves of GRB afterglows are rare. However, a number of robotic telescopes have observed afterglows at times early enough to compare the optical to the high-energy X-ray and $\gamma$-ray emission. As pointed out by Kumar \& Panaitescu (2008), this early-time emission can be divided into three groups: (1) The optical emission traces the high-energy emission (e.g., GRB 041219A; Vestrand et al. 2006); the optical emission does not trace the high-energy emission (e.g., GRB 990123; Akerlof et al. 1999); and (3) the optical emission has two components, one that traces the the high-energy emission and a smoother component, probably the onset of the afterglow, that does not (e.g., GRB 050820A; Vestrand et al. 2006). GRB 0600607A falls into the second of these categories (see $\S 4.1)$.

PROMPT (Reichart et al. 2005) has been designed specifically to observe the prompt optical and near-infrared emission from GRB afterglows simultaneously at multiple wavelengths. The strength of this design is seen in the high-quality, densely sampled light curves of GRB 060607A presented in Figure 1. Early in the afterglow's lifetime, it evolves on a rapid time-scale, and only with simultaneous, multiwavelength observations are we able to properly characterize this phase. With a data set of this quality we are able to 
ask detailed questions about the nature of the early afterglow and explore the possibility of chromatic variations. In this paper, we present the general properties of the afterglow, compare the optical, near-infrared and high-energy emission, and focus on the interpretation of the variability seen in the light curve. In $\S 2$ we review the Swift high-energy observations and present specific details of the PROMPT response. In $\S 3$ we fit the standard afterglow and extinction curve models and discuss the three distinct periods that occur within the first two hundred minutes. In $\S 4$ we compare the optical, near-infrared and Swift x-ray light curves and also discuss the nature of the observed variations. We draw conclusions in $\S 5$.

\section{Observations}

The Swift Burst Alert Telescope (BAT) discovered GRB 060607A at 05:12:13 UT on June $7^{\text {th }}, 2006$ (Ziaeepour et al. 2006). The burst duration is $\mathrm{T}_{90}=102$ seconds $(15-350$ $\mathrm{keV}$ ) (Sakamoto et al. 2007) and exhibits a triply-peaked structure (Tueller et al. 2006). Sixty-five seconds after the initial trigger, the Swift's XRT found a fading x-ray afterglow (Page et al. 2006), and at 75 seconds, UVOT began to observe the bright optical afterglow (Oates et al. 2006). Covino et al. (2006) quickly reported a detection in the near-infrared made by the REM telescope, which began 1.5 minutes after the trigger (Molinari et al. 2006). Nysewander \& Haislip (2006) reported $r$ magnitudes that detailed the rise of the early afterglow. Ledoux et al. (2006), began imaging with VLT 7.5 minutes after the BAT trigger and found an afterglow redshift of $\mathrm{z}=3.082$. Tueller et al. (2006) find that using the observed $15-150 \mathrm{keV}$ fluence of $2.6 \pm 0.1 \times 10^{-6} \mathrm{erg} \mathrm{cm}^{-2}, \mathrm{z}=3.082$, and standard cosmology $\left(\Omega_{M}=0.3, \Omega_{\Lambda}=0.7, \mathrm{H}_{0}=65\right), \mathrm{E}_{i s o}=1.1 \times 10^{53}$ ergs in the rest frame $1-1000$ keV band.

Four 0.4-m PROMPT telescopes began observing the afterglow of GRB 060607A in the Bessell $B$ and SDSS gri filters on June $7^{t h}$, 2006, 05:12:57 UT, 44 seconds after the initial satellite trigger and 25 seconds after the GCN notification. Observations continued for 5.4 hours under the control of Skynet ${ }^{3}$, PROMPT's custom-designed automation software. All images taken after 3.3 hours do not yield detections or limiting magnitudes deep or significant enough to be included in the analysis. Table 1 presents the observations that are plotted in Figure 1. Figure 1 also includes the near-infrared $H$-band light curve measured by the REM telescope (Molinari et al. 2006).

Zero, dark and flat-field calibration images were applied using IRAF's CCDPROC

\footnotetext{
${ }^{3}$ Skynet is a dynamic, prioritized queue-scheduling system that controls a growing number of telescopes, currently spanning North and South America; http://skynet.unc.edu
} 
package, and, if necessary, images were combined to obtain better signal-to-noise. Point-spread function photometry was applied via IRAF's DAOPHOT package to obtain final magnitudes. Zeropoints for each image were measured by reobserving the GRB field along with observing photometric standards with PROMPT on the night of June 12, 2006. Each PROMPT telescope uses a 1k x 1k Apogee Alta U47+ CCD, coated with either a midband or broadband coating in order to maximize the CCD response in the telescope's primary filter set. The cameras have fast-readout technology for fast cadence imaging, with an inter-exposure deadtime of only 2.5 seconds.

\section{Analysis}

Because of the five densely sampled distinct light curves, we are able to see two significant rebrightenings, although additional smaller fluctuations are suggested by the data. Therefore, we model the afterglow as the sum of three peaks, each consisting of a smoothly broken, rising and falling power-law light curve and a simple power-law spectrum, where the spectrum is extinguished by both Milky-Way and source-frame dust and absorbed by hydrogen in the source frame and by the Ly $\alpha$ forest along the line of sight:

$$
F_{\nu}(t)=\sum_{n=1}^{3} e^{-\tau_{\nu}^{M W}} e^{-\tau_{\nu(1+z)}^{L y \alpha}} e^{-\tau_{\nu(1+z)}^{\text {source }}} F_{n}\left(\frac{\nu}{\nu_{o}}\right)^{-\beta_{n}}\left[\left(\frac{t}{t_{n}}\right)^{-s_{n} \alpha_{n, 1}}+\left(\frac{t}{t_{n}}\right)^{-s_{n} \alpha_{n, 2}}\right]^{-1 / s_{n}} .
$$

$\tau_{\nu}^{M W}$ is the Galactic extinction curve model of Cardelli et al. (1989), $\tau_{\nu(1+z)}^{\text {Ly }}$ is the Ly $\alpha$ forest absorption model of Reichart $(2001)$, and $\tau_{\nu(1+z)}^{\text {source }}$ is the source-frame extinction curve and Lyman-limit absorption model of Reichart (2001) taking into account the Ly $\alpha$ dampening wing model of Totani et al. (2006), which is a function of $N_{H} . \alpha_{1 n}$ and $\alpha_{2 n}$ are the rising $\left(\alpha_{1 n}>0\right)$ and falling $\left(\alpha_{2 n}<0\right)$ temporal indices of the $n^{t h}$ peak, $\beta_{n}$ is the spectral index, $t_{n}$ is the peak time, $F_{n}$ is the normalization, $s_{n}$ is the smoothing parameter, and $\nu_{o}$ is the effective frequency of the Sloan $r$ filter. All magnitudes are converted to fluxes as prescribed by Bessell (1979) and Bessell \& Brett (1988). Since the extinction and absorption models have features that are narrower than most photometric bands, we integrate equation (1) against the appropriate filter transmissivity curve before fitting it to the data.

We fit this model to the data using Bayesian inference (e.g., Reichart 2001; Lee et al. 2001; Galama et al. 2003; Nysewander et al. 2006). The posterior probability distribution is equal to the product of the prior probability distribution and the likelihood function. The likelihood function is given by:

$$
\mathcal{L}=\prod_{i=1}^{N} \frac{1}{\sqrt{2 \pi\left(\sigma_{i}^{2}+\sigma^{2}\right)}} \exp \left\{-\frac{1}{2} \frac{\left[y\left(\nu_{i}, t_{i}\right)-y_{i}\right]^{2}}{\sigma_{i}^{2}+\sigma^{2}}\right\},
$$


where $N$ is the number of measurements, $y\left(\nu_{i}, t_{i}\right)$ is the integration of Equation 1 against the spectral curve of the $i$ th measurement at the time of the $i$ th measurement; $y_{i}$ is the $i$ th measurement in units of $\log$ spectral flux; $\sigma_{i}$ is the uncertainty in the $i$ th measurement in the same units, and $\sigma$ is a parameter, sometimes called the slop parameter, that models other, small sources of uncertainty (Reichart 2001). We account for systematic error in zero point calibrations by allowing a magnitude offset for each filter where this offset is constrained by a Gaussian prior of width given by the scatter in the relative magnitudes of the calibration stars. These offsets are small and indicate calibration errors less than 0.1 magnitudes for the PROMPT observations. The calibration offset adopted for the REM H-band light curve is $<0.05$ magnitudes.

Many of the parameters of the source-frame extinction curve model and all of the parameters of the Ly $\alpha$ forest, Galactic extinction curve, and Ly $\alpha$ damping wing models are constrained a priori. The source-frame extinction curve model of Reichart (2001) is a function of eight parameters: the source-frame $\mathrm{V}$-band extinction magnitude $A_{V}$, $R_{V}=A_{V} / E(B-V)$, the intercept $c_{1}$ and slope $c_{2}$ of the linear component of the source-frame UV extinction curve, the strength $c_{3}$, width $\gamma$ and center $x_{o}$ of the UV bump component of the extinction curve, and the strength $c_{4}$ of the FUV excess component of the extinction curve. The Ly $\alpha$ forest absorption model of Reichart (2001) is a function of a $D_{A}$, the flux deficit. Reichart (2001) determines prior probability distributions for $R_{V}, c_{1}, \gamma, x_{o}$, and $D_{A}$, which means that the values of these parameters can be weighted by fairly narrow distributions, the description of which depends on other parameters $\left(c_{2}\right.$ and $\left.z\right)$, a priori. We adopt these priors here. The Galactic extinction curve model of Cardelli et al. (1989) is a function of $E(B-V)=0.029 \mathrm{mag}$ for this line of sight (Schlegel et al. 1998) and a single parameter, $R_{V}^{M W}$. We adopt a prior for this parameter that is log normally distributed with mean $\log 3.1$ and width 0.1, which approximates the distribution of values of this parameter along random lines of through the Galaxy. The Ly $\alpha$ dampening wing model is a function of the density of neutral hydrogen, $N_{H}$, which has a prior of $N_{H}=7.9_{-3.5}^{+3.8} \times 10^{21} \mathrm{~cm}^{-2}$ based on the measurement of Page et al. (2006).

We fit an unconstrained model to the data and then impose relationships between the post-peak temporal and spectral indices of the first component - these relationships depend upon the local environment of the progenitor: a wind-swept (WIND, Chevalier \& Li 2000) or constant density (ISM, Sari et al. 1998) medium, and the placement of the cooling break, $\nu_{c}$, above (BLUE) or below (RED) the observed frequencies. For the ISM-RED and WIND-RED cases, $\alpha_{1,2}=\left(3 \beta_{1}+1\right) / 2=-(3 p-2) / 4$; for the ISM-BLUE case, $\alpha_{1,2}=3 \beta_{1} / 2=-3(p-1) / 4 ;$ and for the WIND-BLUE case, $\alpha_{1,2}=\left(3 \beta_{1}-1\right) / 2=-(3 p-1) / 4$, where $p$ is the power-law index of the electron-energy distribution. In order to test the spectral variability of the second and third components, only $\beta_{1}$ and $\alpha_{1,2}$ are tied together 
using these relationships.

Using the PROMPT Bgri light curves and the REM $H$-band light curve (Molinari et al. 2006), we find that WIND-BLUE with $p=2.12_{-0.07}^{+0.09}$ is the most likely scenario, and is consistent with the general fit at the $2.3 \sigma$ confidence level. The ISM-BLUE and ISM/WIND-RED models are ruled out at the 3.3 and $8.0 \sigma$ confidence levels, respectively; only the WIND-BLUE case is consistent with the observed shallow spectral index $\beta=-0.70$. Given the observed temporal index of $\alpha_{1,2}=-1.34_{-0.07}^{+0.05}$, for ISM-BLUE, $\beta_{1}=-0.89_{-0.05}^{+0.03}$, and for ISM/WIND-RED, $\beta_{1}=-1.23 \pm 0.04$. Often, if a steep spectral slope is observed, degeneracy may exist between a steep intrinsic spectral index and the steepening effect of added extinction, but for GRB 060607A, the observed shallow slope does not allow for ambiguity.

Although we find a small amount of source-frame extinction, $A_{V}=0.41_{-0.30}^{+0.14} \mathrm{mag}$ ( $A_{V}>0$ at the $2.6 \sigma$ credible level), the parameters for the extinction curve model are not well constrained. $c_{1}$ and $R_{V}$ depend upon $c_{2}$, and all are poorly constrained: $c_{1}=-16.2_{-7.3}^{+11.0}$ and $c_{2}=5.5_{-3.3}^{+2.2}$, while $R_{V}$ is completely unconstrained. $c_{3}$ and $c_{4}$ are set to zero because they are second order effects on the global shape of the spectrum. Similarly, although the shape of the light curve is well fit by the model (see Figure 1), the exact fitting parameter values are again degenerate. Because of the extreme slopes involved with the second component, the parameters describing it are not well-defined. For the initial onset of the afterglow, $F_{1}(\mu \mathrm{Jy})=4.32_{-0.14}^{+0.04}, \alpha_{1,1}=2.46_{-0.20}^{+0.23}, \alpha_{1,2}=-1.34_{-0.07}^{+0.05}, \beta_{1}=-0.56_{-0.05}^{+0.03}$, log $t_{1}$ (days) $=-2.70_{-0.02}^{+0.01}$, and $s_{1}=1.72_{-0.38}^{+0.50}$. For the second component, $F_{2}(\mu \mathrm{Jy})>2.83(3 \sigma)$, $\alpha_{2,1}>3.0(3 \sigma), \alpha_{2,2}<-3.6(3 \sigma), \beta_{2}=-0.49_{-0.16}^{+0.15}, \log t_{2}$ (days) $=-1.45_{-0.15}^{+0.08}$, and $s_{2}<6.4$ $(3 \sigma)$. For the final component, $F_{3}(\mu \mathrm{Jy})=2.53_{-0.18}^{+0.20}, \alpha_{3,1}>0.57(2 \sigma), \alpha_{3,2}=-1.28_{-0.20}^{+0.16}$,

$\beta_{3}=-0.16_{-0.24}^{+0.17}, \log t_{3}$ (days) $=-1.36_{-0.09}^{+0.04}$, and $s_{3}>3.29(1 \sigma)$. All values are cited with $1 \sigma$ uncertainties, however, when only an upper or lower limit could be placed, the value is cited with the most constraining limit found $(1,2$, or $3 \sigma)$. Additionally $\log N_{H}=21.69_{-0.21}^{+0.24}$ $\mathrm{cm}^{-2}$, and the slop parameter $\sigma=0.07 \pm 0.01 \mathrm{mag}$.

\section{Discussion}

Three peaks are evident in the prompt $\gamma$-ray emission observed by the Swift BAT: two overlapping FRED profiles from $\mathrm{t}_{0}-5$ to $\mathrm{t}_{0}+40$ seconds and a third component at $\approx 100$ seconds (Tueller et al. 2006). The PROMPT $g$ and $r$ light curves overlap the third $\gamma$-ray peak, and although they are sparsely sampled at this time, we do not observe any obvious corresponding features. Similarly, the peaks in the Swift XRT light curve around 90 and 250 seconds (Molinari et al. 2006) do not appear in the PROMPT observations. 
Furthermore, neither the initial afterglow peak nor the later variations at optical frequencies have corresponding features in the x-ray light curve, although the x-ray light curve at the time of the peaks is not well-sampled. A comparison of the PROMPT $r$ light curve and the Swift XRT x-ray light curve (Evans et al. 2007) is presented in Figure 2. Also, within the early optical and near-infrared light curves themselves, there is no suggestion of chromatic variations.

\subsection{The Early Light Curve}

Both PROMPT and the REM telescope observed the early peak in the afterglow at three minutes. Molinari et al. (2006) attribute this peak to the deceleration of the fireball and in a detailed analysis, use this peak time to calculate the Lorentz factor. Given that the spectral index should be $+1 / 3$ when $\nu_{o p t}<\nu_{m}$ (e.g. Granot \& Sari 2002), the passage of $\nu_{m}$, the synchrotron peak frequency, is ruled out as a cause for the peak, and hence confirms the result of Molinari et al. (2006). Although the pre-peak spectral index may show signs of evolution, the spectral index is not positive (see Figure 1), hence $\nu_{m}<\nu_{\text {opt }}$ before the onset of observations.

The light curve at extreme early times does not appear to be well-fit by the rising emission, but the discrepancies may be due to lower signal-to-noise of the early points. In the very early light curve, deviations from the model exist at the 1.8, 0.9 and $1.4 \sigma$ level for the initial $g, r$ and $i$ points respectively. Early variations in the light curve can be interpreted as being due to activity from the source, however, the early deviations do not correlate with peaks in the X-ray light curve. The lower time resolution of the optical light curves makes it difficult to compare the low and high-energy emission.

Spectral evolution before the peak time is similarly difficult to quantify. The spectral slope measured solely from these three points, centered at 84 seconds is poorly constrained: $\beta=-6.5 \pm 3.3$. The spectral slope measured from all points before the maximum light (during the time period from 65 to 174 seconds) is $\beta=-1.18 \pm 0.52$. This slope is nearly consistent with the over-all slope of $\beta=-0.56_{-0.05}^{+0.02}$ for the first emission component.

\subsection{Comparison with X-Ray Afterglow}

The beginning of the Swift era has brought many new questions about the nature of x-ray afterglows. X-ray light curves often do not correlate with their optical counterparts, even when taking into account spectral breaks between the two frequencies. Possible 
explanations for the disparity include a long-lived central engine that continuously refreshes the forward shock (Zhang et al. 2006), two separate components to produce the x-ray and optical afterglows, or variable microphysical parameters of the shock front (Panaitescu et al. 2006). Given $p=2.12_{-0.07}^{+0.09}$ from the optical light curve, we can examine the correlation between the high and low energy afterglows and determine if they match predictions of the standard model scenarios.

The broadband optical to x-ray spectral flux distribution at twelve minutes after the burst is presented in Figure 3. The x-ray afterglow flux is taken from Molinari et al. (2006) and is plotted using the observed x-ray spectral index $\beta_{x}=-0.64 \pm 0.07$. $\beta_{x}$ was measured from the late-time PC data (which range from 600 seconds to two days), and although the early flares experienced spectral evolution (Page et al. 2006), we assume this value of $\beta_{x}$ for the plot. The SFD is plotted at twelve minutes, which is located immediately after the $\mathrm{x}$-ray flares and near the break in the x-ray light curve when the temporal index shallows from $\alpha_{x 1}=-1.09 \pm 0.04$ to $\alpha_{x 2}=-0.45 \pm 0.03$ (Page et al. 2006). The broadband spectral flux distribution indicates that, at this time, the low and high energy light curves may be correlated. Although uncertain given the temporal scaling and insecure spectral slope, Figure 3 shows the extrapolation of the flux from the x-ray to optical band, which suggests that before the break in the x-ray light curve, one underlying mechanism may be producing both high and low-energy emission.

However, the best fit model to the optical and near-infrared afterglow is one with $\nu_{c}$ blueward of optical frequencies $\left(\nu_{c}>\nu_{\text {opt }}\right.$ at the $8.0 \sigma$ credible level). Contrary to a constant density medium, for the synchrotron spectrum of a shock expanding in a wind-swept environment, $\nu_{c}$ moves upwards through frequency space. At twelve minutes, although $\nu_{c}>\nu_{\text {opt }}, \nu_{c}$ has not likely passed above x-ray frequencies, hence the lack of a break in Figure 3 would be inconsistent with our best fit.

Indeed, the difference in temporal slope at this time $\left(\alpha_{o p t}=-1.34_{-0.07}^{+0.05} \mathrm{vs}\right.$. $\alpha_{x 1}=-1.09 \pm 0.04$ or $\alpha_{x 2}=-0.45 \pm 0.03$ ) indicates that there is a break between the high and low frequencies. Therefore, it is possible that $\beta_{x}$ underwent a change in slope between twelve minutes and when it was measured, and our assumed spectral slope plotted in Figure 3 is incorrect. In the WIND-BLUE fit: $p=2.12_{-0.07}^{+0.09}, \alpha_{\text {opt }}=-1.34_{-0.07}^{+0.05}$ and $\beta_{o p t}=-0.56_{-0.05}^{+0.03}$. If $\nu_{o p t}<\nu_{c}<\nu_{x}$, then the x-ray data will be described by the WIND-RED case and the predicted values for the temporal and spectral x-ray slopes are: $\alpha_{x, W-R}=-1.09_{-0.07}^{+0.05}$ and $\beta_{x, W-R}=-1.06_{-0.05}^{+0.03}$. Note that the predicted value for the x-ray temporal slope matches the observed early x-ray slope of $\alpha_{x, W-R}=\alpha_{x}=-1.09$, hence the pre-break $(t<12$ minutes) period of the x-ray light may be produced by the the same synchrotron component as that of the optical light curve. The break to the shallower, 
$\alpha_{x 2}=-0.45 \pm 0.03$, slope would then correspond to the onset of a second phase of emission not associated with the optical afterglow.

\subsection{Modeling the Variations}

Two clear achromatic rebrightening episodes are observed in the optical and nearinfrared light curves at 40 and 66 minutes. Here we consider possible scenarios for these peaks: the passage of a spectral break, the patchy-shell model, density variations, refreshed shocks, and associated reverse shocks. We do not expect that the variations in the light curve will be due to more than one scenario, as it would be unlikely for multiple conditions to be satisfied (Nakar et al. 2003).

At the time near the peaks of the latter two components, the x-ray light curve is noisy and not well-sampled, however it appears to flatten before the beginning of the first optical rebrightening and remains shallow until the end of the PROMPT light curve before dropping suddenly at $\approx 4$ hours (see Figure 2 ). Because $\nu_{c}$ is likely between the optical and $\mathrm{x}$-ray bands, the lack of features in the high-energy emission may suggest that the episodes are due to density variations in the circumburst medium: variations that depend upon changes in energy would produce variations both above and below $\nu_{c}$. However, without a clear understanding of the nature of the x-ray light curve and the relationship between it and the optical, this claim is questionable. If, as suggested in Section $§ 4.1$, the nearly flat phase of the x-ray emission is not produced by the same mechanism as that of the optical, then evidence for variations above $\nu_{c}$ may be masked by the flux from a second component.

\subsubsection{Spectral Evolution}

We first consider whether the peaks are due to the passage of a break - although the indices are not generally well constrained, they may suggest spectral evolution:

$\beta_{1}=-0.56_{-0.05}^{+0.03}, \beta_{2}=-0.49_{-0.16}^{+0.15}$ and $\beta_{3}=-0.16_{-0.24}^{+0.17}$. Deviations from our simple models of breaks in the electron distribution might influence the synchrotron spectrum and light curve in unexpected ways. A model with no spectral evolution, where we impose that $\beta_{1}=\beta_{3}$ differs from the WIND-BLUE model at only the $1.9 \sigma$ confidence level. The observed change in spectral index $\left(\beta_{3}=\beta_{1}+0.40 \pm 0.18\right)$, from a moderate to shallow slope, is roughly consistent with the passage of $\nu_{c}$ from low to high frequencies. However, the WIND and ISM-RED models are ruled out for the early data at the $8.0 \sigma$ confidence level, and hence we do not find this to be a likely scenario. 


\subsubsection{Patchy Shells}

The patchy-shell model, where the observed flux depends upon the angular structure of the jet, has variations with a timescale longer than the observed time, $\Delta t>t$, and more specifically, has decay times, $t_{\text {decay }} \approx t$ (Nakar \& Oren 2004). At the end of the fluctuations, the light curve will fade to match the extrapolation of the power-law light curve before the episode occurred. The timescale for the first variation is short, $\Delta t \approx 27 \mathrm{~min}$, but the peak of the variation is at $33 \mathrm{~min}$, therefore the first relation is marginally satisfied. However,

the first peak decays rapidly, $t_{\text {decay }} \approx 15 \mathrm{~min}$, which is too extreme to be expected from the timescale of the observations. The second variation may satisfy both of these relationships; however, the post-peak decay index does not indicate that the light curve is fading to its pre-episode flux. Therefore, because the first variation is not consistent with the predicted constraints and the decay of the second variation matches the pre-episode index, the patchy shell model is also unlikely.

\subsubsection{Density Variations}

Variations due to density enhancements in the circumburst environment can inflate the flux of the light curve that then settles into the afterglow solution describing the new environment. Generally, for a single density fluctuation, the light curve will asymptotically relax to the pre-episode brightness. However, if the shock impacts a shell after which the density increases permanently, then it is possible for the light curve to appear to renormalize. In addition to this, as in the patchy-shell scenario, it is difficult for even a very sharp fluctuation in the density to produce short time-scale fluctations in the light curve (Nakar \& Piran 2003) with $\Delta t<t$. Therefore, we do not find it likely that the first of the two variations is produced via this scenario.

However, if the jump in density is high enough ( 21, Dai \& Lu 2002), then a reverse shock may form in addition to the enhancement of the forward shock. Due to the rapidly fading nature of the second feature, we find this to be a plausible explanation for the second episode. In this scenario, though, the apparent renormalization after the second variation is a coincidence not predicted by the model, and relies upon conditions specific to the circumburst density profile. 


\subsubsection{Energy Injection}

If the bumps are due to two episodes of energy injection, either by the continuing activity of the progenitor or by late internal shocks resulting from slower shells impacting the main forward shock, then the fading temporal $\left(\alpha_{1,2}=\alpha_{2,2}=\alpha_{3,2}\right)$ and spectral $\left(\beta_{1}=\beta_{2}=\beta_{3}\right)$ indices will remain nearly constant. The energy injection will raise the flux of the light curve and although the post-shock indices will reflect the new shock conditions, they should vary little from the previous state. Due to the steepness of $\alpha_{4}$ this model is ruled out at the $7.5 \sigma$ confidence level.

However, as in the case of density variations, it is possible that internal shocks can cause a bright reverse shock to form and be observed as a bright, rapidly fading flare in the GRB afterglow. In this case, the first and third variations will have nearly the same indices $\left(\alpha_{1,2}=\alpha_{3,2} ; \beta_{1}=\beta_{3}\right)$ and the second variation will fade rapidly $\left(\alpha_{2,2} \geq 2\right)$. Quantitatively, this model is consistent with the general model at the $1.9 \sigma$ uncertainty level. Qualitatively, this is precisely what we see: the second episode at 66 minutes is a simple renormalization after which the afterglows fades with the same initial slope, whereas the first clearly fades more steeply. In fact, focusing on the pre- and post-episode slopes, a model with $\alpha_{1,2}=\alpha_{3,2}$ is consistent with a model where the parameters are unconstrained at the $0.4 \sigma$ confidence

level. Therefore, we find that a refreshed shock that was preceeded by a bright reverse shock is the most likely cause of the two rebrightening episodes. The observations are consistent with density variations, however, the renormalization required in that circumstance would be merely coincidental, rather than a prediction of the model.

\section{Conclusions}

We have presented the early-time PROMPT afterglow light curve for GRB 060607A, fit a model to the data and found that the progenitor likely exploded into a wind-swept medium and that the cooling frequency of the synchrotron spectrum is above the optical frequencies before the beginning of the PROMPT observations. Observing correlations between peaks in different energy ranges is a key tool for probing the underlying physics of the central engine and its interaction with its environment. Although the x-ray spectrum exhibited intense flares at early times, these flares are not present in the optical light curves. Likewise, optical flares at later times are not observed in the x-ray light curve. In the PROMPT optical light curve we see an initial peak at 2.9 minutes, and later, two clear rebrightening episodes at 40 and 66 minutes that are not observed at high energies. We do not observe spectral evolution throughout the PROMPT dataset and constrain $\nu_{m}$ to be below and $\nu_{c}$ to be above optical frequencies by the onset of observations. 
Many authors have cited the need for observations at both low and high energy in order to distinguish between variations due to energy or density, and in this case the lack of features in the high-energy light curve would indicate that the fluctations are due to density. However, without a clear understanding of the mechanisms that produce the light curves, we cannot definitively relate them. We find that the most likely scenario for the observed fluctuations is that of energy injection to the forward shock, either through a delayed shock or continuing activity of the central engine, which is strong enough to produce a bright reverse shock that sweeps through the relativistic material behind the main forward shock. In the case of GRB 060607, although energy injection is the favored scenario, the constraints imposed by the light curves are not conclusive proof.

As efforts to observe the afterglows of $\gamma$-ray bursts have strengthened, data sets grow more robust. Clearly, if variations are ubiquitous, the analysis of a poorly sampled single light curve is difficult to trust. The results obtained via modeling such a light curve that has undergone episodes of rebrightening will likely deviate from its true behavior, and the steepness of temporal decays will be systematically underestimated. PROMPT is a powerful tool that can produce densely sampled early-time light curves when afterglows are bright and more likely to undergo rapid variations. By observing simultaneously in multiple filters, we can test the early-time afterglow for chromatic variations, measure spectral indices, and obtain multiple, independent light curves. Because of these factors, PROMPT will play a strong role in future afterglow follow-up studies.

DER very gratefully acknowledges support from NSF's MRI, CAREER, AAG, and PREST programs, NASA's APRA, Swift GI, and IDEAS programs, NC Space Grant's NIP program, and especially Leonard Goodman and Henry Cox. We gratefully acknowledge Don Smith for useful comments and collaboration on the PROMPT project, and also T. Brennan, M. Schubel and J. Styblova for their work as new members of the UNC GRB Follow-Up Group. This work made use of data supplied by the UK Swift Science Data Centre at the University of Leicester. 
Table 1. PROMPT Observations of the Afterglow of GRB 060607A

\begin{tabular}{|c|c|c|c|c|}
\hline Mean Time (UT) & Exp. Time & Mean $\Delta \mathrm{t}(\mathrm{hr})$ & Filter & Magnitude \\
\hline Jun 7 5:13:38 & $15 \mathrm{~s}$ & 0.0235 & $B$ & $>16.61$ \\
\hline Jun 7 5:14:09 & $20 \mathrm{~s}$ & 0.0322 & $B$ & $16.44 \pm 0.10$ \\
\hline Jun 7 5:14:40 & $20 \mathrm{~s}$ & 0.0408 & $B$ & $15.69 \pm 0.07$ \\
\hline Jun 7 5:15:11 & $20 \mathrm{~s}$ & 0.0494 & $B$ & $15.47 \pm 0.06$ \\
\hline Jun 7 5:15:42 & $20 \mathrm{~s}$ & 0.0581 & $B$ & $15.63 \pm 0.07$ \\
\hline Jun 7 5:16:25 & $40 \mathrm{~s}$ & 0.0700 & $B$ & $15.59 \pm 0.04$ \\
\hline Jun 7 5:17:15 & $40 \mathrm{~s}$ & 0.0839 & $B$ & $15.84 \pm 0.05$ \\
\hline Jun 7 5:18:06 & $40 \mathrm{~s}$ & 0.0981 & $B$ & $16.03 \pm 0.06$ \\
\hline Jun 7 5:18:56 & $40 \mathrm{~s}$ & 0.1119 & $B$ & $16.21 \pm 0.07$ \\
\hline Jun 7 5:20:07 & $80 \mathrm{~s}$ & 0.1317 & $B$ & $16.51 \pm 0.09$ \\
\hline Jun 7 5:21:45 & $80 \mathrm{~s}$ & 0.1589 & $B$ & $16.55 \pm 0.05$ \\
\hline Jun 7 5:23:23 & $80 \mathrm{~s}$ & 0.1861 & $B$ & $16.86 \pm 0.06$ \\
\hline Jun 7 5:25:01 & $80 \mathrm{~s}$ & 0.2133 & $B$ & $17.16 \pm 0.11$ \\
\hline Jun 7 5:26:36 & $80 \mathrm{~s}$ & 0.2397 & $B$ & $17.25 \pm 0.12$ \\
\hline Jun 7 5:28:53 & $160 \mathrm{~s}$ & 0.2778 & $B$ & $17.60 \pm 0.09$ \\
\hline Jun 7 5:32:44 & $240 \mathrm{~s}$ & 0.3420 & $B$ & $17.77 \pm 0.09$ \\
\hline Jun 7 5:37:26 & $240 \mathrm{~s}$ & 0.4203 & $B$ & $17.89 \pm 0.08$ \\
\hline Jun 7 5:42:04 & $240 \mathrm{~s}$ & 0.4974 & $B$ & $17.85 \pm 0.07$ \\
\hline Jun 7 5:46:36 & $240 \mathrm{~s}$ & 0.5730 & $B$ & $17.75 \pm 0.08$ \\
\hline Jun 7 5:51:50 & $320 \mathrm{~s}$ & 0.6601 & $B$ & $17.98 \pm 0.07$ \\
\hline Jun 7 6:00:52 & $640 \mathrm{~s}$ & 0.8110 & $B$ & $18.56 \pm 0.09$ \\
\hline Jun 7 6:14:19 & $800 \mathrm{~s}$ & 1.0350 & $B$ & $18.61 \pm 0.11$ \\
\hline Jun 7 6:28:11 & $640 \mathrm{~s}$ & 1.2662 & $B$ & $18.91 \pm 0.11$ \\
\hline Jun 7 7:09:32 & $960 \mathrm{~s}$ & 1.9553 & $B$ & $19.53 \pm 0.19$ \\
\hline Jun 7 7:48:25 & $1200 \mathrm{~s}$ & 2.6033 & $B$ & $19.98 \pm 0.18$ \\
\hline Jun 7 8:18:06 & $1360 \mathrm{~s}$ & 3.0981 & $B$ & $19.75 \pm 0.14$ \\
\hline Jun 7 5:13:20 & $5 \mathrm{~s}$ & 0.0185 & $g$ & $16.63 \pm 0.15$ \\
\hline Jun 7 5:13:46 & $10 \mathrm{~s}$ & 0.0258 & $g$ & $16.11 \pm 0.10$ \\
\hline Jun 7 5:14:46 & $10 \mathrm{~s}$ & 0.0425 & $g$ & $15.04 \pm 0.03$ \\
\hline
\end{tabular}

${ }^{\mathrm{a}}$ Upper limits are $3 \sigma$. 
Table 2. PROMPT Observations of the Afterglow of GRB 060607A (con`t)

\begin{tabular}{lcccc}
\hline \hline Mean Time $(\mathrm{UT})$ & Exp. Time & Mean $\Delta \mathrm{t}(\mathrm{hr})$ & Filter & Magnitude \\
\hline Jun $75: 15: 48$ & $20 \mathrm{~s}$ & 0.0597 & $g$ & $15.09 \pm 0.03$ \\
Jun 7 5:17:17 & $20 \mathrm{~s}$ & 0.0844 & $g$ & $15.37 \pm 0.02$ \\
Jun 7 5:19:03 & $40 \mathrm{~s}$ & 0.1139 & $g$ & $15.82 \pm 0.02$ \\
Jun 7 5:21:55 & $80 \mathrm{~s}$ & 0.1617 & $g$ & $16.10 \pm 0.01$ \\
Jun 7 5:25:06 & $80 \mathrm{~s}$ & 0.2147 & $g$ & $16.67 \pm 0.03$ \\
Jun 7 5:29:45 & $80 \mathrm{~s}$ & 0.2922 & $g$ & $17.20 \pm 0.03$ \\
Jun 7 5:31:17 & $80 \mathrm{~s}$ & 0.3178 & $g$ & $17.30 \pm 0.05$ \\
Jun 7 5:35:57 & $80 \mathrm{~s}$ & 0.3956 & $g$ & $17.38 \pm 0.04$ \\
Jun 7 5:37:29 & $80 \mathrm{~s}$ & 0.4211 & $g$ & $17.31 \pm 0.04$ \\
Jun 7 5:42:11 & $80 \mathrm{~s}$ & 0.4994 & $g$ & $17.27 \pm 0.04$ \\
Jun 7 5:43:42 & $80 \mathrm{~s}$ & 0.5247 & $g$ & $17.36 \pm 0.04$ \\
Jun 7 5:45:17 & $80 \mathrm{~s}$ & 0.5511 & $g$ & $17.33 \pm 0.04$ \\
Jun 7 5:46:27 & $40 \mathrm{~s}$ & 0.5706 & $g$ & $17.37 \pm 0.07$ \\
Jun 7 5:54:12 & $80 \mathrm{~s}$ & 0.6997 & $g$ & $18.04 \pm 0.06$ \\
Jun 7 5:55:42 & $80 \mathrm{~s}$ & 0.7247 & $g$ & $18.04 \pm 0.07$ \\
Jun 7 5:57:13 & $80 \mathrm{~s}$ & 0.7500 & $g$ & $18.05 \pm 0.05$ \\
Jun 7 5:58:24 & $40 \mathrm{~s}$ & 0.7697 & $g$ & $18.15 \pm 0.07$ \\
Jun 7 6:08:25 & $280 \mathrm{~s}$ & 0.9367 & $g$ & $18.52 \pm 0.17$ \\
Jun 7 6:26:04 & $240 \mathrm{~s}$ & 1.2307 & $g$ & $18.41 \pm 0.08$ \\
Jun 7 6:30:39 & $240 \mathrm{~s}$ & 1.3071 & $g$ & $18.41 \pm 0.05$ \\
Jun 7 7:05:40 & $240 \mathrm{~s}$ & 1.8907 & $g$ & $19.20 \pm 0.17$ \\
Jun 7 7:42:28 & $640 \mathrm{~s}$ & 2.5042 & $g$ & $19.48 \pm 0.06$ \\
Jun 7 8:22:50 & $800 \mathrm{~s}$ & 3.1768 & $g$ & $19.74 \pm 0.08$ \\
Jun 7 5:13:18 & $10 \mathrm{~s}$ & 0.0181 & $r$ & $15.98 \pm 0.31$ \\
Jun 7 5:13:44 & $10 \mathrm{~s}$ & 0.0253 & $r$ & $15.75 \pm 0.16$ \\
Jun 7 5:14:43 & $20 \mathrm{~s}$ & 0.0417 & $r$ & $14.34 \pm 0.03$ \\
Jun 7 5:15:45 & $20 \mathrm{~s}$ & 0.0589 & $r$ & $14.32 \pm 0.03$ \\
Jun 7 5:17:19 & $40 \mathrm{~s}$ & 0.0850 & $r$ & $14.68 \pm 0.02$ \\
Jun 7 5:19:01 & $40 \mathrm{~s}$ & 0.1133 & $r$ & $15.11 \pm 0.02$ \\
\hline
\end{tabular}


Table 3. PROMPT Observations of the Afterglow of GRB 060607A (con`t)

\begin{tabular}{|c|c|c|c|c|}
\hline Mean Time (UT) & Exp. Time & Mean $\Delta \mathrm{t}(\mathrm{hr})$ & Filter & Magnitude \\
\hline Jun 7 5:21:47 & $80 \mathrm{~s}$ & 0.1594 & $r$ & $15.47 \pm 0.02$ \\
\hline Jun 7 5:25:03 & $80 \mathrm{~s}$ & 0.2139 & $r$ & $16.04 \pm 0.03$ \\
\hline Jun 7 5:29:43 & $80 \mathrm{~s}$ & 0.2917 & $r$ & $16.60 \pm 0.05$ \\
\hline Jun 7 5:31:12 & $80 \mathrm{~s}$ & 0.3164 & $r$ & $16.57 \pm 0.05$ \\
\hline Jun 7 5:35:56 & $80 \mathrm{~s}$ & 0.3953 & $r$ & $16.57 \pm 0.04$ \\
\hline Jun 7 5:37:26 & $80 \mathrm{~s}$ & 0.4203 & $r$ & $16.75 \pm 0.08$ \\
\hline Jun 7 5:42:09 & $80 \mathrm{~s}$ & 0.4989 & $r$ & $16.69 \pm 0.07$ \\
\hline Jun 7 5:43:38 & $80 \mathrm{~s}$ & 0.5236 & $r$ & $16.60 \pm 0.06$ \\
\hline Jun 7 5:45:11 & $80 \mathrm{~s}$ & 0.5494 & $r$ & $16.64 \pm 0.08$ \\
\hline Jun 7 5:46:20 & $40 \mathrm{~s}$ & 0.5686 & $r$ & $16.78 \pm 0.12$ \\
\hline Jun 7 5:54:10 & $80 \mathrm{~s}$ & 0.6992 & $r$ & $17.25 \pm 0.08$ \\
\hline Jun 7 5:55:39 & $80 \mathrm{~s}$ & 0.7239 & $r$ & $17.37 \pm 0.08$ \\
\hline Jun 7 5:57:09 & $80 \mathrm{~s}$ & 0.7489 & $r$ & $17.37 \pm 0.10$ \\
\hline Jun 7 5:58:38 & $80 \mathrm{~s}$ & 0.7736 & $r$ & $17.54 \pm 0.12$ \\
\hline Jun 7 6:08:24 & $280 \mathrm{~s}$ & 0.9365 & $r$ & $17.65 \pm 0.11$ \\
\hline Jun 7 6:28:15 & $480 \mathrm{~s}$ & 1.2672 & $r$ & $17.69 \pm 0.06$ \\
\hline Jun 7 7:05:04 & $320 \mathrm{~s}$ & 1.8810 & $r$ & $18.44 \pm 0.08$ \\
\hline Jun 7 7:41:40 & $720 \mathrm{~s}$ & 2.4909 & $r$ & $18.77 \pm 0.09$ \\
\hline Jun 7 8:23:22 & $880 \mathrm{~s}$ & 3.1859 & $r$ & $19.04 \pm 0.15$ \\
\hline Jun 7 5:14:12 & $20 \mathrm{~s}$ & 0.0331 & $i$ & $14.75 \pm 0.05$ \\
\hline Jun 7 5:15:14 & $20 \mathrm{~s}$ & 0.0503 & $i$ & $14.05 \pm 0.04$ \\
\hline Jun 7 5:16:27 & $40 \mathrm{~s}$ & 0.0706 & $i$ & $14.20 \pm 0.04$ \\
\hline Jun 7 5:18:10 & $40 \mathrm{~s}$ & 0.0992 & $i$ & $14.76 \pm 0.05$ \\
\hline Jun 7 5:20:13 & $80 \mathrm{~s}$ & 0.1333 & $i$ & $15.01 \pm 0.04$ \\
\hline Jun 7 5:23:25 & $80 \mathrm{~s}$ & 0.1867 & $i$ & $15.61 \pm 0.05$ \\
\hline Jun 7 5:26:38 & $80 \mathrm{~s}$ & 0.2403 & $i$ & $15.99 \pm 0.07$ \\
\hline Jun 7 5:28:07 & $80 \mathrm{~s}$ & 0.2650 & $i$ & $16.23 \pm 0.08$ \\
\hline Jun 7 5:32:49 & $80 \mathrm{~s}$ & 0.3433 & $i$ & $16.34 \pm 0.10$ \\
\hline Jun 7 5:34:18 & $80 \mathrm{~s}$ & 0.3681 & $i$ & $16.43 \pm 0.08$ \\
\hline
\end{tabular}


Table 4. PROMPT Observations of the Afterglow of GRB 060607A (con't)

\begin{tabular}{lcccc}
\hline \hline Mean Time (UT) & Exp. Time & Mean $\Delta \mathrm{t}(\mathrm{hr})$ & Filter & Magnitude \\
\hline Jun $75: 39: 02$ & $80 \mathrm{~s}$ & 0.4469 & $i$ & $16.48 \pm 0.08$ \\
Jun $75: 40: 31$ & $80 \mathrm{~s}$ & 0.4717 & $i$ & $16.53 \pm 0.07$ \\
Jun $75: 48: 08$ & $80 \mathrm{~s}$ & 0.5986 & $i$ & $16.68 \pm 0.09$ \\
Jun $75: 49: 37$ & $80 \mathrm{~s}$ & 0.6233 & $i$ & $16.60 \pm 0.11$ \\
Jun $75: 51: 08$ & $80 \mathrm{~s}$ & 0.6486 & $i$ & $16.79 \pm 0.10$ \\
Jun $76: 00: 27$ & $400 \mathrm{~s}$ & 0.8038 & $i$ & $17.33 \pm 0.07$ \\
Jun $76: 15: 52$ & $480 \mathrm{~s}$ & 1.0609 & $i$ & $17.46 \pm 0.07$ \\
Jun $77: 13: 22$ & $560 \mathrm{~s}$ & 2.0190 & $i$ & $18.32 \pm 0.09$ \\
Jun $78: 03: 47$ & $720 \mathrm{~s}$ & 2.8594 & $i$ & $18.94 \pm 0.19$ \\
\hline
\end{tabular}


Fig. 1.- The Bgri afterglow of GRB 060607A from 44 seconds to 3.2 hours taken with the PROMPT telescopes along with the $H$-band REM light curve (Molinari et al. 2006). The solid lines present the best fit to the initial peak at three minutes and two variations that occur at 42 and 64 minutes.

Fig. 2.- The PROMPT $r$-band light curve plotted with the XRT x-ray afterglow scaled to units of $10^{4}$ counts. Note that the time of the variations in the optical correspond to a lapse in x-ray observations. However, the x-ray light curve appears to flatten around this time and hence does not undergo the same fluctuating behavior as the optical light curve.

Fig. 3. - The broadband spectral flux distribution of the afterglow of GRB 060607A plotted at twelve minutes. The solid lines are the fit to the data; the dotted lines are the unextinguished model. The high energy x-ray flux is adopted from Molinari et al. (2006), and the plotted spectral index is taken from Page et al. (2006). 


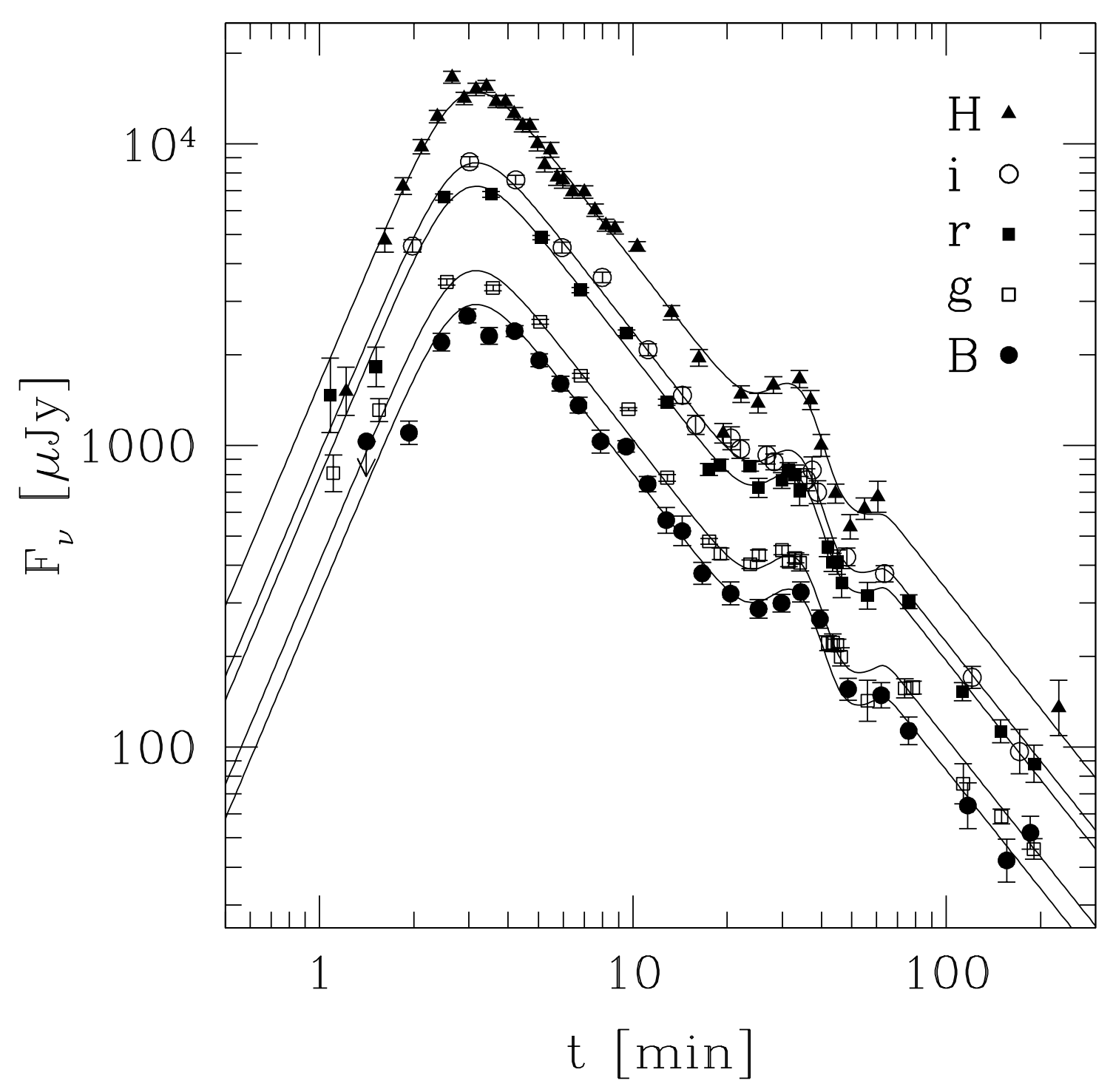




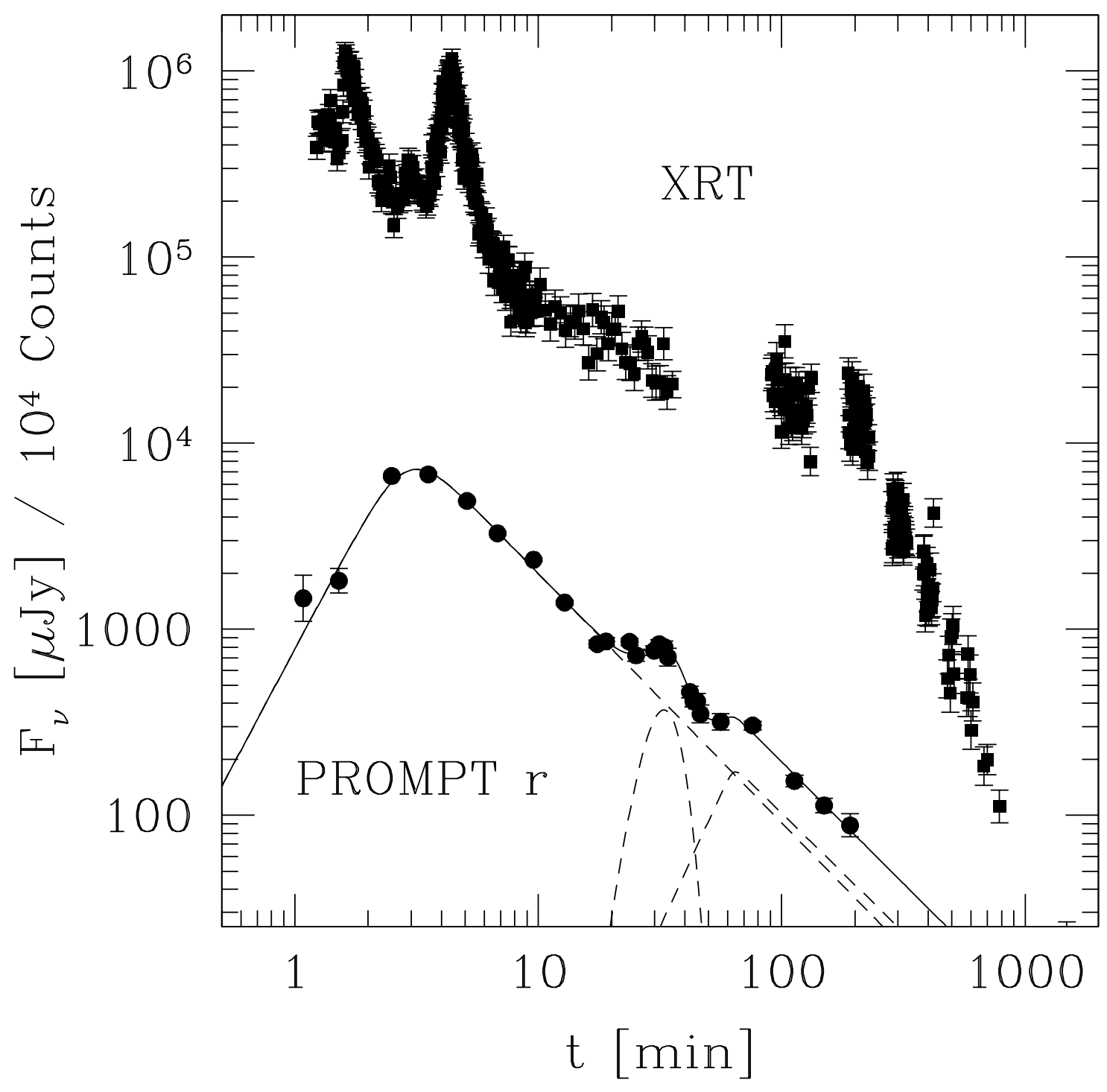




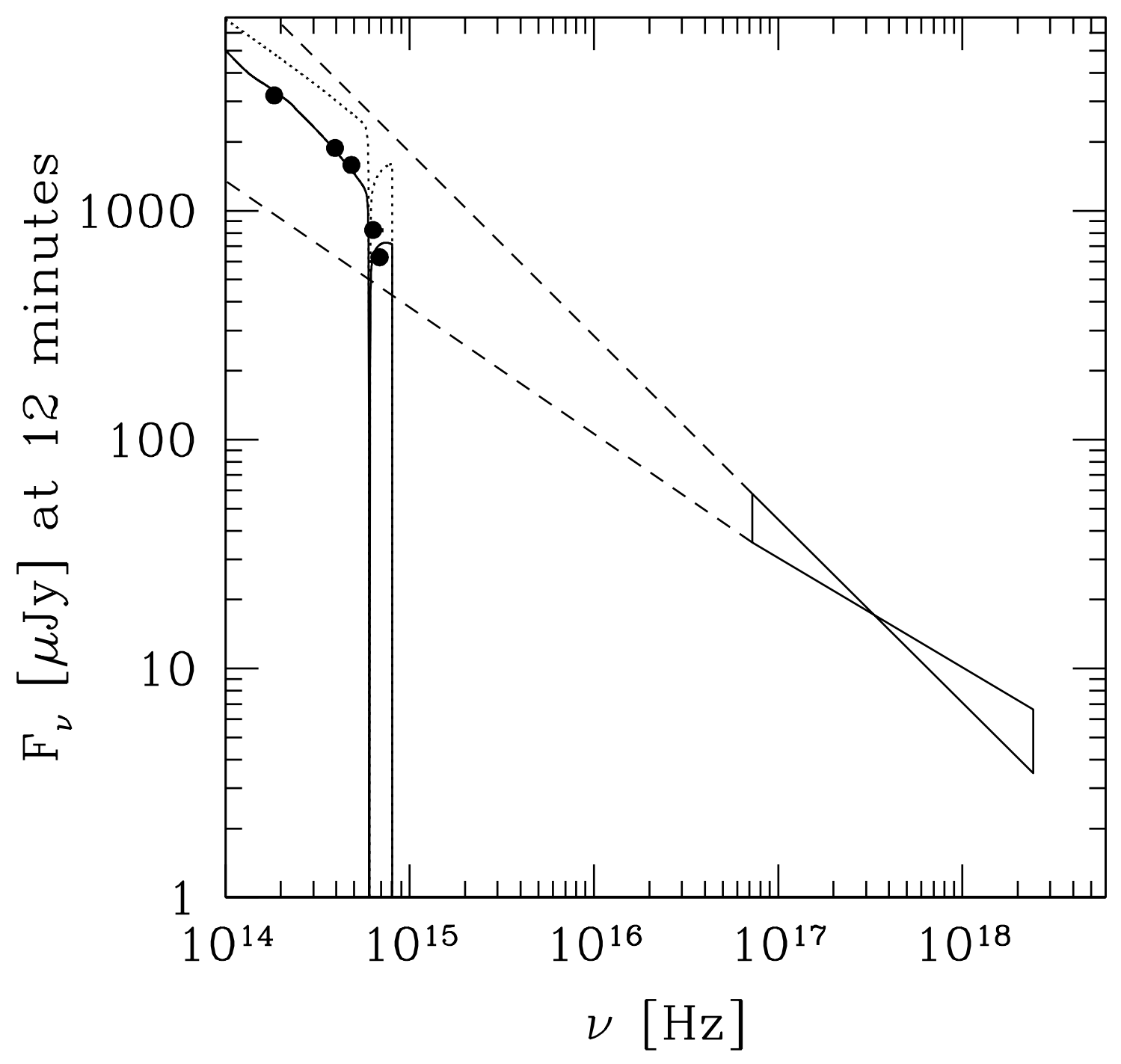




\section{References}

Bessell, M. S. 1979, PASP, 91, 589

Bessell, M. S., \& Brett, J. M. 1988, PASP, 100, 1134

Björnsson, G., Gudmundsson, E. H., \& Jóhannesson, G. 2004, ApJ, 615, L77

Cardelli, J. A., Clayton, G. C., \& Mathis, J. S. 1989, ApJ, 345, 245

Chevalier, R. A., \& Li, Z.-Y. 2000, ApJ, 536, 195

Costa, E. et al. 1997, Nature, 387, 783

Covino, S. et al. 2006, GRB Coordinates Network, 5234, 1

Dai, Z. G., \& Lu, T. 2002, ApJ, 565, L87

de Ugarte Postigo, A. et al. 2005, Astronomy \& Astrophysics, 443, 841

Evans, P. A. et al. 2007, ArXiv e-prints, 704

Fox, D. W. et al. 2003, Nature, 422, 284

Frail, D. A., Kulkarni, S. R., Nicastro, S. R., Feroci, M., \& Taylor, G. B. 1997, Nature, 389, 261

Galama, T. J. et al. 2003, ApJ, 587, 135

Granot, J., Nakar, E., \& Piran, T. 2003, Nature, 426, 138

Granot, J., \& Sari, R. 2002, ApJ, 568, 820

Huang, Y. F., Cheng, K. S., \& Gao, T. T. 2006, ApJ, 637, 873

Kumar, P., \& Panaitescu, A. 2008, ArXiv e-prints, 805

Kumar, P., \& Piran, T. 2000a, ApJ, 535, 152

-. 2000b, ApJ, 532, 286

Lazzati, D., Rossi, E., Covino, S., Ghisellini, G., \& Malesani, D. 2002, Astronomy \& Astrophysics, 396, L5

Ledoux, C., Vreeswijk, P., Smette, A., Jaunsen, A., \& Kaufer, A. 2006, GRB Coordinates Network, 5237, 1 
Lee, B. C. et al. 2001, ApJ, 561, 183

Molinari, E. et al. 2006, ArXiv Astrophysics e-prints

Nakar, E., \& Oren, Y. 2004, ApJ, 602, L97

Nakar, E., \& Piran, T. 2003, ApJ, 598, 400

Nakar, E., Piran, T., \& Granot, J. 2003, New Astronomy, 8, 495

Nysewander, M., \& Haislip, J. 2006, GRB Coordinates Network, 5236, 1

Nysewander, M. C. et al. 2006, ApJ, 651, 994

Oates, S. R., Blustin, A. J., \& Ziaeepour, H. Z. 2006, GRB Coordinates Network, 5243, 1

Page, K., Goad, M., \& Beardmore, A. 2006, GRB Coordinates Network, 5240, 1

Panaitescu, A., Mészáros, P., Burrows, D., Nousek, J., Gehrels, N., O’Brien, P., \& Willingale, R. 2006, MNRAS, 369, 2059

Panaitescu, A., Meszaros, P., \& Rees, M. J. 1998, ApJ, 503, 314

Rees, M. J., \& Meszaros, P. 1998, ApJ, 496, L1+

Reichart, D. et al. 2005, Nuovo Cimento C Geophysics Space Physics C, 28, 767

Reichart, D. E. 2001, ApJ, 553, 235

Sakamoto, T. et al. 2007, ArXiv e-prints, 707

Sari, R., \& Mészáros, P. 2000, ApJ, 535, L33

Sari, R., Piran, T., \& Narayan, R. 1998, ApJ, 497, L17+

Schlegel, D. J., Finkbeiner, D. P., \& Davis, M. 1998, ApJ, 500, 525

Totani, T., Kawai, N., Kosugi, G., Aoki, K., Yamada, T., Iye, M., Ohta, K., \& Hattori, T. 2006, Publications of the ASJ, 58, 485

Tueller, J. et al. 2006, GRB Coordinates Network, 5242, 1

Uemura, M. et al. 2004, PASJ, 56, 77

van Paradijs, J. et al. 1997, Nature, 386, 686

Wang, X., \& Loeb, A. 2000, ApJ, 535, 788 
Zhang, B., Fan, Y. Z., Dyks, J., Kobayashi, S., Mészáros, P., Burrows, D. N., Nousek, J. A., \& Gehrels, N. 2006, ApJ, 642, 354

Zhang, B., \& Mészáros, P. 2002, ApJ, 566, 712

Ziaeepour, H. Z. et al. 2006, GRB Coordinates Network, 5233, 1 